\title{
La revitalización de la lengua embera en Colombia: de la oralidad a la escritura ${ }^{12}$
}

The revitalization of Embera language in

Colombia: from orality to literacy

\section{Andoni Barreña Agirrebeitia \\ Universidad de Salamanca \\ España}

Número especial

Las lenguas amerindias en Iberoamérica: retos para el siglo XXI

2017
ONOMÁZEIN - Número especial

Las lenguas amerindias en Iberoamérica: retos para el siglo XXI (2017): 58-76 DOI: 10.7764/onomazein.amerindias.03

\section{(C) $\underset{\mathrm{BY}}{(\mathrm{i})}$}

Andoni Barreña Agirrebeitia: Universidad de Salamanca, España

| Correo electrónico: barrena.andoni@gmail.com

Manel Pérez-Caurel: Cátedra UNESCO de Patrimonio Lingüístico Mundial, Universidad del País Vasco/Euskal Herriko Unibertsitatea UPV/EHU, España. | Correo electrónico: manel.caurel@ehu.eus 


\section{Resumen}

Mientras que el pueblo embera había rechazado codificar su lengua, a finales del siglo XX los embera del Chocó (Colombia) decidieron utilizar la escritura y facilitar así su introducción en la escuela. Fue en 2010 cuando se inició un proyecto de tres años con este objetivo. Este artículo describe, en primer lugar, los datos generales sobre la lengua y la sociedad embera; en segundo lugar, las características generales del proyecto y el estado de desarrollo del mismo. Finalmente, se plantean los retos que se vislumbran para el mantenimiento del embera en el futuro inmediato.

Palabras clave: lengua embera; cooperación lingüística; estandarización; revitalización; capacitación de docentes indígenas.

\section{Abstract}

While the Embera people had in the past rejected to codify its own language, at the end of the 2oth century the Embera from Chocó (Colombia) decided to start using a writing system for their language so as to introduce it in education. It was in 2010 when a three-year project was designed to accomplish this task. This article describes, firstly, the general background of the Embera language and society; secondly, it deals with the features of the project and its state of development. Finally, the article addresses the challenges the future poses to the maintenance of the Embera.

Keywords: Embera language; linguistic cooperation; language standardization; revitalization; training of indigenous teachers.

1 Este proyecto de cooperación contó con financiación de la Agencia Española de Cooperación Internacional para el Desarrollo (AECID) en el periodo 2010-2011 y de la Viceconsejería de Política Lingüística del Gobierno Vasco en 2012.

2 Exceptuando los mapas, cuya fuente es citada, las imágenes incluidas en este artículo fueron tomadas por Andoni Barreña durante el desarrollo del proyecto. 


\section{Introducción}

La gran mayoría de pueblos del mundo cuya lengua entró en un proceso de pérdida presenta también carencias y muestras de pobreza en otros ámbitos. En el caso de muchos pueblos indígenas dicha pérdida es evidente, dado que la mera asistencia económica no soluciona los problemas que menguan el bienestar social integral y el desarrollo personal de sus miembros. En las reservas de los pueblos originarios de los Estados Unidos se observa que, además de no superar la pobreza, esta situación da lugar a la aparición y a la prevalencia de situaciones de exclusión y marginación en muchos casos.

En un estudio publicado a comienzos de este milenio (véase Martí y otros, 2005) se recogía información y opinión de 525 comunidades lingüísticas diferentes de todo el planeta; los informantes reconocían que sus comunidades lingüísticas consideraban totalmente necesaria la presencia de sus lenguas en el sistema escolar y reclamaban, en los casos en que la presencia era nula o limitada, una integración total de la misma. En el continente americano los movimientos indígenas reclaman, desde hace al menos 30 años, que sus lenguas sean utilizadas y respetadas en todos los ámbitos sociales, muy especialmente en la enseñanza (López y Sichra, 2008; Sichra, 2009). También demandan que la escuela indígena sea creada desde y para la cultura y cosmovisión indígenas.

En el marco de la política estatal de Colombia se viene trabajando por potenciar el respeto por las manifestaciones propias del lenguaje de cada uno de los pueblos indígenas que sobreviven en el país (el Senado de la República de Colombia aprobó en último debate el proyecto de Ley de Lenguas Nativas el 9 de diciembre de 2009). En lo que se refiere a la necesidad de formación académica de los indígenas, históricamente se han desarrollado diferentes planes en los que la educación impartida se llevaba a cabo primordialmente desde el castellano.

En este artículo se pretende informar sobre la experiencia que se está llevando a cabo desde el año 2010 en el Chocó colombiano con el fin de incorporar la lengua y cultura emberas al sistema escolar indígena y contribuir así a la conservación de la diversidad etnolingüística.

\section{El pueblo embera y su lengua}

El pueblo embera constituye uno de los grupos indígenas más importantes que habitan la región del Pacífico colombiano. Este grupo indígena se ha ubicado en una gran extensión de territorio, desde el suroccidente de Centroamérica a lo largo del noroccidente suramericano. Ha ampliado sus territorios tradicionales por medio de sucesivas migraciones, y por el norte ha alcanzado la provincia del Darién en el litoral pacífico panameño; hacia el sur ocupa las cabeceras de los ríos Naya, Saija, Satinga y Sanquianga hasta la provincia de Esmeraldas, en Ecuador. Hacia el este los embera han traspasado en tiempos recientes la cordillera, ubicán- 
dose en los departamentos del Caquetá y del Putumayo. Más concretamente, las comunidades embera se encuentran desde la provincia del Darién en Panamá, en los departamentos colombianos de Antioquía, Córdoba, Caldas, Chocó, Risaralda, Valle, Cauca y Nariño, hasta Ecuador (establecidos fundamentalmente en los ríos Naya, Yurumangié, San Juan, Atrato y el Sijai).

La lengua embera o emberá3, hablada por alrededor de 70000 indígenas, forma parte de la familia lingüística chocó, que comprende también el wounaan o waunana, aparte de los distintos dialectos embera (Aguirre, 1999). La mayoría de los embera vive en Colombia; cerca de 20000 en Panamá y unos cuantos cientos en Ecuador. En Colombia la mayoría de los emberahablantes (cerca de 52 000) se concentra en el departamento del Chocó, pero también hay comunidades embera en Antioquia, Bolívar, Caldas, Córdoba, Risaralda, Valle, Cauca y Nariño.

\section{MAPA 1}

Ubicación geográfica del pueblo embera marcada en color rosa (s. f.)

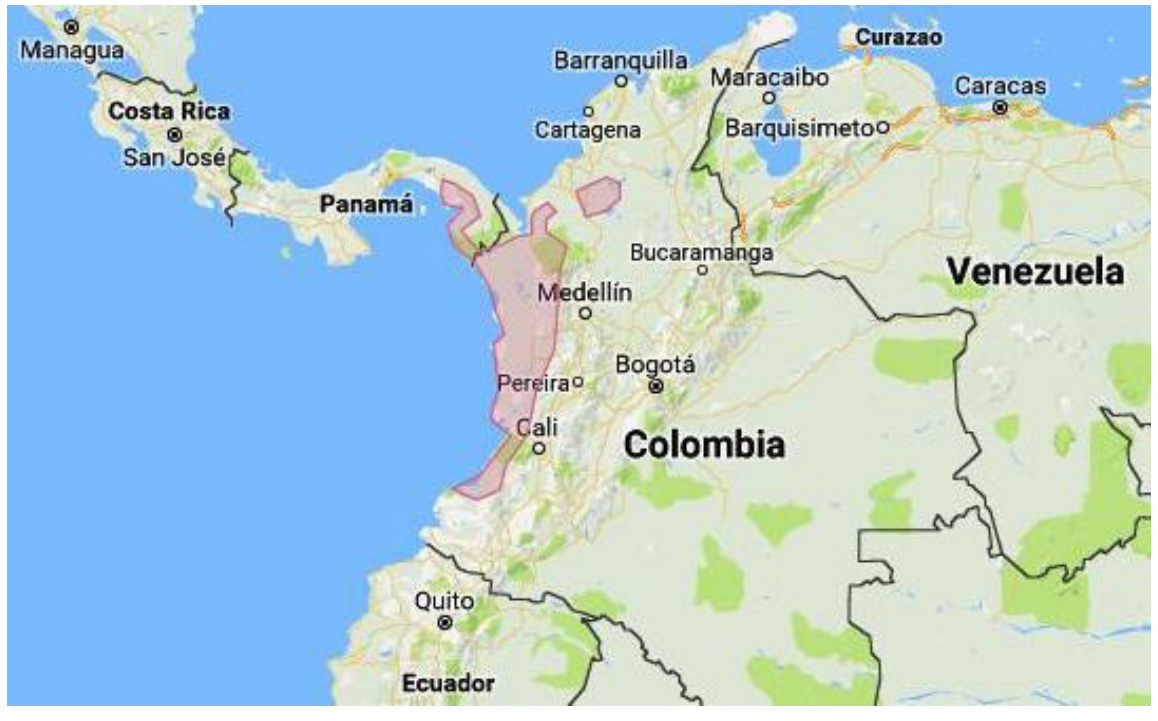

En este grupo étnico la mayoría es hablante de la lengua propia. No obstante, la situación social y lingüística de los embera de Colombia ha cambiado drásticamente en los últimos años, debido a que la población civil de esta zona ha sufrido — sobre todo las mujeres y los niños - constantemente las graves consecuencias humanitarias del conflicto armado, principalmente el desplazamiento forzado. Por ello, actualmente es posible encontrar numerosa

3 En inglés y castellano se le conoce como lengua embera. En embera se escribe ēpera (en la propuesta de escritura del Chocó) o ēbēra (en la propuesta de escritura de Antioquia). 
población embera en ciudades como Medellín y Bogotá, sin que se haya realmente estudiado el impacto que este fenómeno ha causado a nivel social y lingüístico.

El pueblo embera está compuesto por varios grupos que comparten su historia, tradiciones y cosmovisión. Sin embargo, la lengua nativa de cada uno de los grupos que lo componen difiere y presenta variedades dialectales en proceso de ser unificadas. Los estudios sobre la lengua y la sociedad emberas son muy limitados; los propios embera trabajan con la hipótesis de que su lengua la componen cuatro dialectos diferenciados pero altamente comprensibles entre sí: el topida (en castellano embera dobida4; to, 'río'; topida, gentilicio de lugar, 'gente de río'), eyapida o eapida (en castellano embera katío o embera eyabida; eya o ea, 'monte'; eyapida, 'gente de montaña, montañeses'), oipida (en castellano embera chamí o embera oibida; oi, 'selva'; oipida, 'gente de selva') y eperarã siapidarã (sia, 'flecha'; siapida, 'gente de flecha’). Aunque antiguamente las variedades embera se diferenciaban geográficamente, en la actualidad, debido a los desplazamientos forzosos de muchas de sus comunidades, comparten el mismo territorio o se encuentran próximas.

La lengua y la sociedad embera han sido descritas por lingüistas y antropólogos colombianos y extranjeros (Loewen, 1963; Pinto García, 1978; Pardo, 1985, 1986, 1987; Pardo y Aguirre, 1993; Llerena Villalobos, 1992, 1994; Aguirre, 1992, 1993, 1995, 1999; Chaves, Ulloa, Morales y Ceron, 1992; Mortensen, 1999; Hoyos Benítez, 2000; Arellano y Arellano, 2002; Cayo Atienza, 2002; Fabre, 2005), pero las descripciones hechas por los propios embera apenas han sido publicadas 5 .

\section{Los embera del Chocó colombiano}

La población indígena en Chocó sigue sufriendo un alto índice de discriminación por parte del resto de la población por el hecho de ser indígenas. Su dificultad de acceso a la educación superior tiene un claro correlato en el elevado fracaso escolar, explicado, en gran parte, tanto por la ausencia de su lengua materna en la enseñanza como por la existencia de barreras económicas. Hay carencias materiales en servicios públicos, salud, educación y energía que afectan por igual a mujeres y hombres embera. En el Chocó los embera constituyen el 9\% de la población del departamento y gestionan 117 resguardos con una extensión de 1497134 hectáreas. La extensión de los territorios gestionados por los resguardos ha ido disminuyen-

4 En el sistema ortográfico del embera los grafemas t y $p$, como se verá a continuación, se acercan a la realización fonológica representada en castellano con las letras $d$ y $b$, lo que provoca que un castellanohablante traslade al castellano de este modo dichos términos.

5 A finales de 2012 los propios embera del Chocó y estos investigadores tuvieron conocimiento de la recogida de material léxico realizada por Borja, un embera eyabida de Antioquia, en 2009. 
do progresivamente debido a los desplazamientos forzosos provocados por los paramilitares y otras fuerzas armadas que han obligado a las comunidades embera a dejar sus territorios ancestrales. Junto a los embera conviven en el Chocó los wounaan —cerca de 5000- unos cientos de gunadules o tules y las comunidades afrocolombianas que constituyen el 90\% de la población del departamento.

Los embera del Chocó conservan su lengua y cultura tradicionales muy vivas. Es posible que algunos embera desplazados voluntaria o forzosamente a entornos urbanos hayan podido olvidar su lengua, pero lo observado por nuestros investigadores, tanto en el entorno urbano de Quibdó, capital del Chocó, como en las comunidades rurales, demuestra que el embera pedea es su lengua habitual, incluso para comunidades no alejadas de redes de comunicación importantes, en las que las mujeres y los niños tienen una competencia muy limitada del castellano (Mecha, Barreña y Mendizabal, 2013).

En 1979 se creó la OREWA como organización estudiantil en defensa de la cultura y de los derechos educativos de los pueblos indígenas en las cabeceras municipales, y ya en 1981 se reformó la Organización Regional Embera Wounaan (Rojas Curieux, 20096), que posteriormente se fragmentó. Son las organizaciones indígenas las que gestionan la escasa autonomía de las comunidades indígenas, entre las que cabe resaltar la del sistema escolar; en el Chocó este proceso se dio a partir de 2011.

Diferentes sectores y líderes de la comunidad embera del Chocó rechazaron hasta 1986 el uso de la escritura fonológica7. Según fue transmitido por miembros de esta comunidad indígena a los investigadores vascos Andoni Barreña y Mikel Mendizabal, este posicionamiento tenía su raíz en el desprestigio y señalamiento de la lengua embera como lengua diabólica por parte de la Iglesia católica al inicio de su intervención civilizatoria, evangelizadora y castellanizadora. Por ello, el rechazo de la enseñanza de la lengua embera a otras culturas se convirtió en una forma de resistencia lingüística y cultural. Pero, a partir de 1986, los embera decidieron iniciarse en la escritura con el objetivo de fortalecer su educación propia y facilitar así a los niños embera el aprendizaje de la lectoescritura desde su propia lengua. Así, hasta 1992 se permitió la investigación lingüística a antropólogos y lingüistas como Mauricio Pardo Rojas, Rito Llerena Villalobos, Mario Hoyos, Martha Espinel y Miller. A partir de ese año, el

6 Los embera participan en las siguientes organizaciones indígenas departamentales: en Antioquia, junto a los gunadule (o tule), en la Organización Indígena de Antioquia (OIA); en el Cauca, con otros 7 pueblos indígenas, en el Consejo Regional Indígena del Cauca (CRIC); en Caldas en el Consejo Regional Indígena de Caldas (CRIDEC); en el Valle en la Oganización Regional Indígena del Valle del Cauca (ORIVAC). Todas estas organizaciones participan en la Organización Nacional Indígena de Colombia (ONIC).

7 Los embera rechazan el uso del término "ágrafo" para su situación anterior, ya que tradicionalmente han representado sus mitos y cultura mediante diseños y dibujos. 
trabajo lingüístico lo asumió Baltazar Mecha Forastero, indígena embera, que el 3 de mayo de 2010 presentó el mandato de la unificación de la escritura de la lengua embera del Chocó, ratificado en la asamblea de Autoridades Indígenas.

En la actualidad, los niños y jóvenes del Chocó se escolarizan principalmente en casteIlano, aunque los docentes en la escuela utilizan oralmente el embera. Con anterioridad a 2011 la diócesis del Chocó gestionaba el sistema escolar indígena y durante los últimos años fomentó la contratación de docentes emberahablantes. No obstante, la inmensa mayoría de los docentes carecen de una capacitación pedagógica académica y, a partir de 2011, son las organizaciones indígenas las que gestionan el sistema escolar indígena.

Ante esta situación, con el propósito de aplicar la unificación de la escritura de la lengua embera y formar a los docentes en la lectoescritura en embera, en 2010 dirigentes educativos de OREWA junto a la Universidad Claretiana FUCLA idearon un proyecto para introducir la lengua embera en el sistema escolar indígena.

\section{Características del proyecto}

La primera redacción del proyecto "Fortalecimiento del uso de la lengua nativa en el proceso de educación escolar para las comunidades embera en el departamento del Chocó”, realizada en 2010, fijó los objetivos del mismo y los productos que se preveía elaborar.

Objetivos:

- Desarrollar una propuesta piloto de educación que asuma la lengua tradicional embera como medio de educación escolar.

- Presentar una propuesta de unificación del alfabeto y formas de escritura embera.

Productos:

- Identificación de la grafía común.

- Identificación de las diferencias de grafía.

- Toma de consenso sobre grafía común y general de la lengua embera.

- Diseño de instrumentos pedagógicos para la enseñanza de la lectoescritura embera.

- Diseño del proceso pedagógico para la estructuración de la gramática embera y su posterior proceso de enseñanza escolar.

Para desarrollar este proceso se propuso un plan de acción de tres años de duración en el que se trabajaría sobre las siguientes cuestiones: investigación y consenso sobre la grafía; elaboración de instrumentos pedagógicos de lectoescritura, y estructuración de la gramática y su proceso pedagógico de enseñanza escolar. 
Es en este punto cuando los promotores del proyecto decidieron solicitar a la Cátedra UNESCO de Patrimonio Lingüístico Mundial de la Universidad del País Vasco acompañamiento para desarrollar el proyecto. La elección del acompañamiento vasco se debió a la experiencia acumulada por el pueblo vasco en el proceso de normalización de su lengua y en la incorporación de la misma al sistema escolar (Garabide, 2010; Mecha, Barreña y Mendizabal, 2013).

\subsection{Justificación del proyecto}

El desarrollo sostenible requiere la participación de todos los grupos, especialmente de las minorías etnolingüísticas que dependen de ecosistemas saludables para su supervivencia y futuro. Sin embargo, la distancia de las minorías de los centros de poder y de las lenguas asociadas a la toma de decisiones (lenguas hegemónicas), las hace especialmente vulnerables. Además, tal y como se avanzó anteriormente, la ausencia de las lenguas autóctonas minorizadas en el sistema escolar es uno de los principales factores que explican el fracaso escolar en los países en vías de desarrollo.

La unificación del sistema de escritura embera permite ofrecer una educación escolarizada a los miembros de las comunidades; significa, además, hacer valer sus derechos, su voz y sus tradiciones ante el resto de las comunidades e instancias sociales, desde sus propias condiciones e ideas. También significa preservar y difundir adecuadamente su pensamiento sin mediaciones que alteren o transformen sus ideas y pensamientos, $y$, fundamentalmente, significa conservar la herencia de que son portadores desde la oralidad, permanecer en el tiempo de acuerdo a lo que su propia lengua dice de sí mismos, no a partir de traducciones o interpretaciones.

La experiencia muestra que los materiales escolares que se usan en las comunidades indígenas (generalmente en lengua dominante) carecen de interés (no han sido elaborados en función de sus necesidades) y apenas contribuyen a la recuperación y desarrollo de las lenguas indígenas. Sabemos por propia experiencia (vasca) que la elaboración de material escolar en lengua propia no dominante es un proceso largo y costoso pero que contribuye directamente a la mejora del sistema educativo. Es decir, no solo asegura el uso de la lengua minorizada, sino que contribuye a adoptar una postura crítica ante dichos materiales.

El uso escrito de la lengua embera facilitará una mayor y más adecuada integración del pueblo embera en la sociedad actual, manteniendo y desarrollando sus raíces culturales. Además, permitirá a los embera acercarse a la cultura y sociedad colombianas, e incluso a la global, respetando y conservando su propia cultura. Si el proceso se realiza de modo equilibrado e integral, los frutos serán más positivos tanto para los embera como para todos los colombianos. 
De cualquier modo, la construcción del proceso debe realizarla la comunidad embera. Son los docentes embera y todos los sectores implicados en el desarrollo del sistema educativo los que deben diseñar y construir los instrumentos pedagógicos necesarios. Quizás, para estas labores, al inicio necesiten el acompañamiento externo, pero el esfuerzo y el protagonismo han de ser del pueblo embera.

\subsection{Metodología del proyecto}

La investigación, la participación y el trabajo en equipo fueron las bases de la metodología del proyecto. El origen del proyecto está en la propia sociedad embera, la verdadera protagonista, particularmente los docentes y la comunidad educativa. Para implementar el proyecto se adoptó un método basado en la realización de talleres de docentes abiertos con un ritmo de trabajo mensual. En dichos talleres se trabajaba en la alfabetización de sus participantes, en la recogida de material léxico y en el diseño de materiales pedagógicos. Los talleres se realizaban coordinados por las autoridades educativas de la organización indígena OREWA y contaron con el acompañamiento de los miembros de la Cátedra UNESCO de la UPV/EHU en algunas ocasiones (los investigadores Andoni Barreña y Mikel Mendizabal).

\subsection{Desarrollo y evaluación del proyecto}

El proyecto se inició en 2010. En un primer momento, se llevó a cabo una reunión preliminar entre los responsables embera y vascos para establecer las líneas generales de actuación; un taller con los docentes embera en el que se trabajó principalmente en torno a tres actividades: la propuesta del sistema ortográfico para el embera, el método a seguir para la recogida de vocabulario y la confección de materiales escolares en embera; y una sesión de evaluación posterior de los responsables.

\subsubsection{La propuesta del sistema ortográfico}

Los responsables embera del proyecto, especialmente Baltazar Mecha, líder de la organización indígena OREWA y uno de los dirigentes del área educativa, presentaron una propuesta de sistema ortográfico que fue aprobada con alguna pequeña modificación. La propuesta representaba gráficamente 12 vocales y 20 consonantes. De las 12 vocales, 6 son orales y las otras 6 nasales (tabla 1). De las 20 consonantes, 8 son oclusivas, 3 nasales, 3 líquidas, 2 africadas, 1 fricativa, 1 aspirada y 2 semiconsonantes (tabla 2). En este punto es necesario precisar que los lingüistas que han descrito el sistema fonológico del embera no coinciden al determinar el número de fonemas consonánticos de la lengua. Por ejemplo, Aguirre (1999) señala que son 16, mientras que Cayo (2002), quien se basa en el trabajo de Loewen (1963), apunta 21. Así 
pues, existen diversas fuentes, pero escasez de estudios realizados por los propios hablantes. El trabajo de Borja (2009), embera de la variedad eyapida del departamento de Antioquia, es una excepción. Él señala la existencia de 19 consonantes.

Los diferentes sistemas ortográficos del Chocó, el propuesto por Baltazar Mecha y aceptado por las Autoridades Indígenas y el seguido para la recogida léxica de Borja (2009), se diferencian en dos aspectos: a) el propuesto por Mecha se basa en 8 consonantes oclusivas, mientras que el utilizado por Borja representa únicamente 7; b) Mecha propone utilizar el dígrafo ts para un fonema realizado como africado en las variantes topida y oipida y como fricativo en la variante eyapida, mientras que Borja propone el grafema $z$, al tener realización fricativa en su dialecto.

\section{TABLA 1}

Las vocales del embera representadas de acuerdo a la propuesta ortográfica de Mecha, de la organización OREWA

\begin{tabular}{|c|c|c|c|}
\hline & ANTERIORES & CENTRALES & POSTERIORES \\
\hline orales altas & i & $4^{8}$ & u \\
\hline orales medias & e & & 0 \\
\hline oral baja & & a & \\
\hline nasales altas & $\tau$ & $\tilde{t}^{9}$ & $\tilde{u}$ \\
\hline nasales medias & $\tilde{e}$ & & $\tilde{0}$ \\
\hline nasal baja & & $\tilde{a}$ & \\
\hline
\end{tabular}

\section{TABLA 2}

Las consonantes del embera representadas de acuerdo a la propuesta ortográfica de Mecha, de la organización OREWA

\begin{tabular}{|c|c|c|c|c|c|c|}
\hline & BILABIALES & DENTALES & ALVEOLARES & PALATALES & VELAR & GLOTAL \\
\hline oclusivas sonoras & b & & $d$ & & g & \\
\hline oclusivas sordas & $p$ & $\mathrm{t}$ & & k & & \\
\hline oclusivas sordas aspiradas & ph & th & & & & \\
\hline vibrante simple & & & $r$ & & & \\
\hline vibrante múltiple & & & $\mathrm{rr}$ & & & \\
\hline continuas nasales & $\mathrm{m}$ & & $n$ & $\tilde{n}$ & & \\
\hline continuas fricativas & & & s & ts & & j \\
\hline continuas africadas & & & & ch & & \\
\hline continuas líquidas & w & । & $y$ & & & \\
\hline
\end{tabular}


La recogida del vocabulario y la elaboración de materiales escolares llevadas a cabo dentro del proyecto se realizaron con la propuesta ortográfica avalada por OREWA.

\subsubsection{La recogida de vocabulario}

En este primer taller de 2010 y en la posterior sesión de evaluación se decidió realizar la recogida de léxico mediante el estudio de diferentes campos semánticos o centros de interés: partes del cuerpo, casa, territorio, familia y organización social, actividades, tiempo atmosférico, tiempo cronológico, adjetivos, sentidos, vestidos y adornos, elementos tecnológicos, enfermedades y procesos biológicos, sistema numérico y operaciones, coquetería, medios de transporte, mitología y lenguaje infantil.

Desde este primer taller se estuvo trabajando en la recogida y sistematización de material léxico en las tres variantes del Chocó, pero esta actividad también servía para la alfabetización en embera de los propios docentes y para el conocimiento de la variación dialectal. Además, la importancia de esta labor fue primordial, ya que, salvo la recogida de material léxico de Borja (2009) en la variante eyapida (de la que no se tuvo conocimiento hasta finales de 2012), los emberahablantes no habían realizado ningún trabajo semejante hasta entonces.

En los posteriores talleres realizados en 2011 y 2012 los docentes embera continuaron recogiendo terminología de los centros de interés arriba mencionados.

\subsubsection{La confección de materiales escolares}

Desde el inicio mismo del proyecto se observó que la alfabetización de los docentes y su formación pedagógica debían constituir los elementos centrales del proyecto. Por ello, en 2010 solo se perfilaron algunos de los materiales escolares que se iban a realizar, pero no se inició la confección de ninguno de ellos. En 2011 se inició la elaboración de dos materiales escolares básicos: el ēpera pedea y el ēpera pedea phãdai' ('alfabeto ilustrado' y 'diccionario ilustrado', respectivamente).

El inicio de la capacitación en la confección de materiales escolares en 2011 se complicó a causa de un paro armado convocado por la guerrilla en el Chocó, que bloqueó las comunicaciones y no permitió a los docentes embera trasladarse a los talleres realizados en la capital

8 Corresponde a/ $\mathrm{t} /$ en AFl: vocal oral cerrada central redondeada.

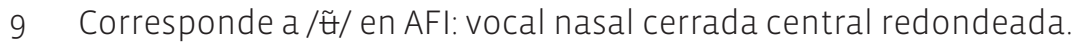

10 Se decidió nombrar en embera los materiales y las actividades realizadas. 
del Chocó, Quibdó. Aún así, durante el 2011, en esta línea de actuación, se inició la elaboración de ambos materiales. Hubo que hacerlo manualmente, ya que entonces los docentes embera no disponían de equipos informáticos. Se elaboraron tres ēpera pedea, uno en cada dialecto (figura 1), y se inició la recogida de material para la confección del ēpera pedea phãdai.

\section{FIGURA 1}

Primera representación del ēpera pedea en la variante topida, referida a la letra e

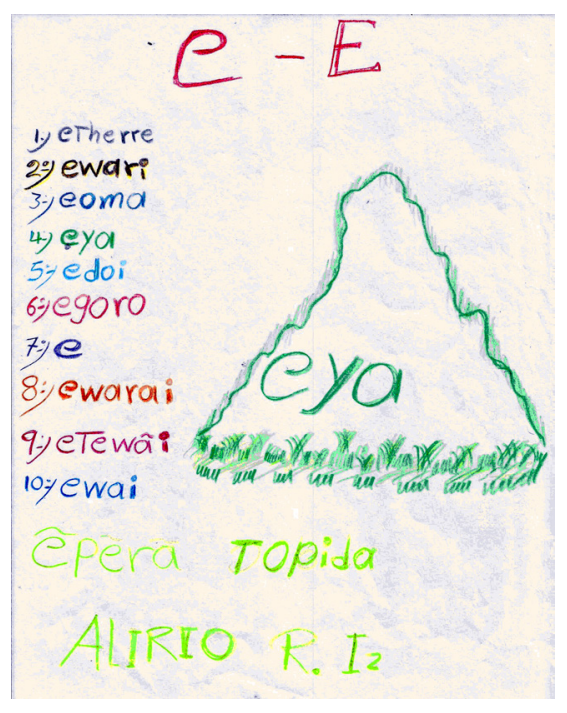

\subsubsection{Evaluación de las medidas de planificación lingüística emprendidas}

A finales de 2011, se realizó una sesión de evaluación conjunta entre los responsables embera, los docentes embera, Ios acompañantes de la Cátedra (Andoni Barreña y Mikel Mendizabal) y los responsables de la Universidad Claretiana FUCLA de Quibdó. La evaluación del trabajo realizado hasta ese momento se consideró positiva por diferentes motivos:

- el proyecto iniciado suponía un salto cualitativo en la actitud de los emberahablantes respecto al uso escrito de su lengua, ya que ahora la reclamaban para el uso escolar, especialmente los jóvenes embera;

- todos los participantes en la evaluación pedían continuar el trabajo por el camino emprendido;

- los docentes embera proponían la participación en el proceso de un mayor número de docentes indígenas, que, según ellos, también lo reclamaban;

- todos los sectores consideraban que era necesaria y posible una planificación de la creación de material escolar en lengua embera;

- todos los sectores proponían continuar con el acompañamiento vasco, como hasta el momento. 
Por ello, a finales de 2011, se diseñó un curso de capacitación y formación específico para los docentes embera, organizado conjuntamente por la organización indígena OREWA y la Universidad Claretiana FUCLA y que contaba con el acompañamiento de la Cátedra UNESCO de Patrimonio Lingüístico Mundial de la UPV/EHU. Se denominó Diplomatura de Etnolingüística y consistía en un curso de 200 horas de trabajo distribuido en 3 semanas alternas de 40 horas de trabajo cada una, además de 80 horas de trabajo personal.

Los objetivos fundamentales del curso fueron los siguientes:

- alfabetización de los docentes embera en su lengua;

- capacitación de los docentes en la creación de materiales escolares básicos en embera;

- concienciación y sensibilización sobre el valor individual y social del uso, cultivo y desarrollo de las lenguas y culturas originarias.

La metodología debía basarse en una capacitación inminentemente práctica, pero sin obviar cuestiones teóricas.

\subsubsection{La capacitación intensiva de los docentes embera}

El curso Diplomatura de Etnolingüística se desarrolló a lo largo de 2012 y fueron 60 docentes embera los que lo realizaron, aunque para ello tuvieran que asumir el coste de la matrícula, los desplazamientos y la estancia en Quibdó durante tres semanas. Algunos de los matriculados en el curso habían participado con anterioridad en los talleres, pero la mayoría de los matriculados no se había iniciado con anterioridad en la escritura del embera. Además, de los 60, solo 6 manejaban equipos informáticos.

Desde el inicio del curso se tomó la determinación de promover el uso de la lengua embera en el mismo, a pesar de que algunos de los docentes la desconocían. Fue, por tanto, la primera ocasión en que la lengua embera fue vehicular en una enseñanza universitaria de modo sistemático y, por ello, se determinó que todas las actividades que se desarrollasen adoptasen el nombre embera y que los docentes solo escribiesen en dicha lengua. En el curso, además de las correspondientes sesiones teóricas, se realizaron numerosos trabajos y talleres prácticos. En todo caso, es necesario remarcar que el objetivo principal no era la elaboración de materiales escolares, sino la capacitación de los docentes en la confección de los mismos.

En la figura 2 se muestran algunas de las actividades emprendidas y trabajos realizados.

Este ejercicio consistió en la realización de un cartel del alfabeto ilustrado del embera en las tres variantes del Chocó. Para ello, como se muestra en la figura 2, se representaron en un cartel todos los fonemas mediante sus grafemas correspondientes, acompañados del dibujo 
de un objeto que los incluía en su denominación. El ejercicio se realizó mediante dibujos manuales que fueron digitalizados para configurar el cartel.

\section{FIGURA 2}

Épera pedea, cartel del alfabeto ilustrado en la variante topida

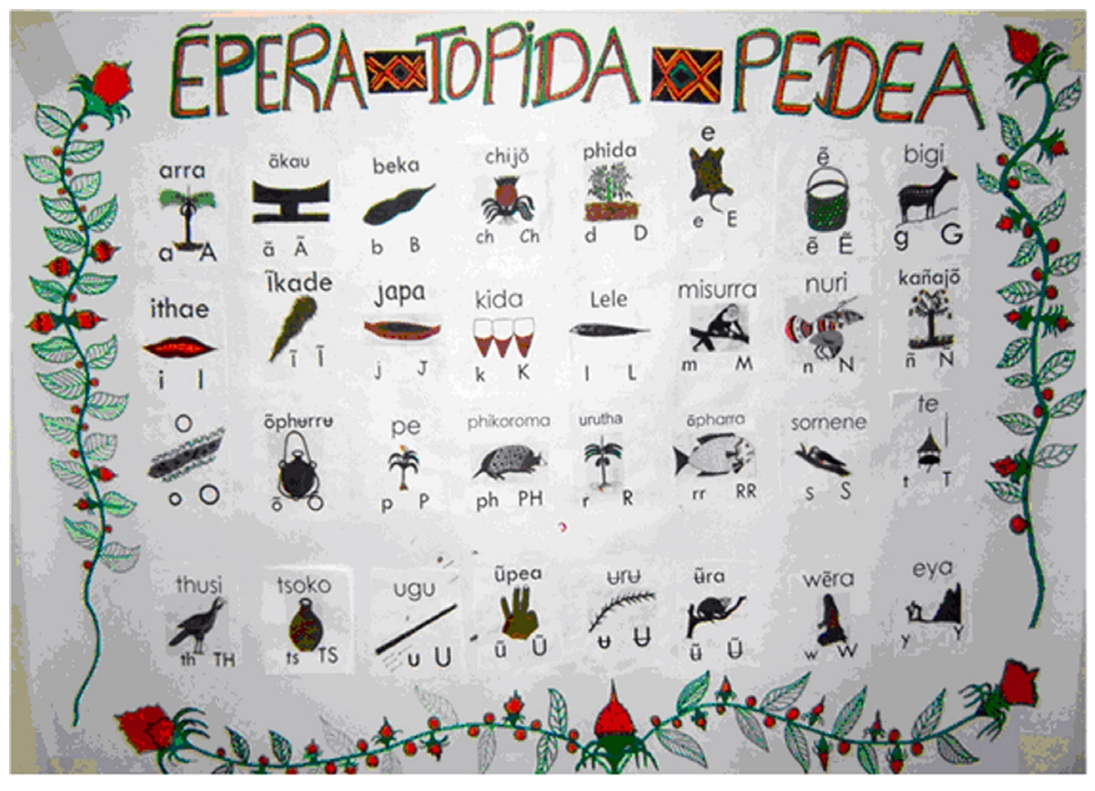

\section{FIGURA 3}

Ne ãrimararã tsake, representación de los insectos con su correspondiente denominación en la variedad eapida o eyapida
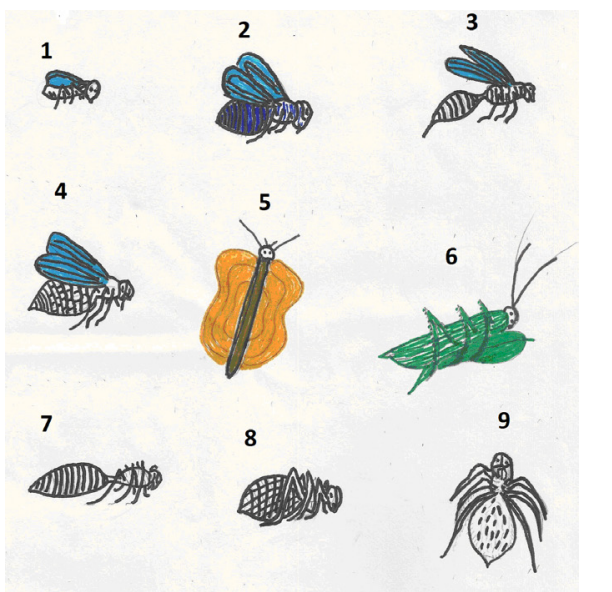

ãrimararã tsake chi buru wēakede

8

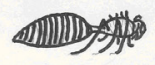

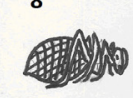

I kũkusi, 2 ukana,

3 neturr, 4 sita,

5 põpõ, 6 sisi,

7 jēsera, 8 ã-ãri,

9 puru, 10 tusõre,

11 thãthã, 12 surra

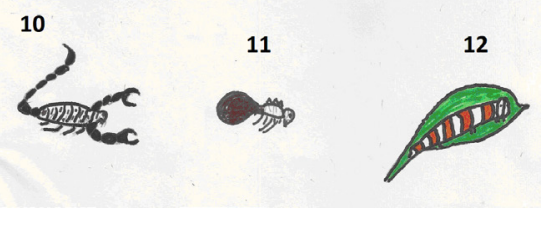


Tal y como se observa en la figura 3, otro de los materiales de capacitación de los docentes embera fue la versión de un diccionario ilustrado en su lengua. Para ello, los docentes recogieron dibujos realizados por ellos mismos que representaban su medio social y natural inmediato. Se trata de un tipo de material clásico en las iniciativas de incorporación de lenguas a la escuela. En la figura 3 se puede ver una recopilación de dibujos referentes a los insectos. Obsérvese que los dibujos han sido digitalizados y el objetivo es crear una base de datos informatizada que pueda ser enriquecida y transformada en futuras versiones.

\section{FIGURA 4}

Ewaritsa bupatat, un ejemplo del cuaderno o diario de un participante en el curso de Diplomatura de Etnolingüística

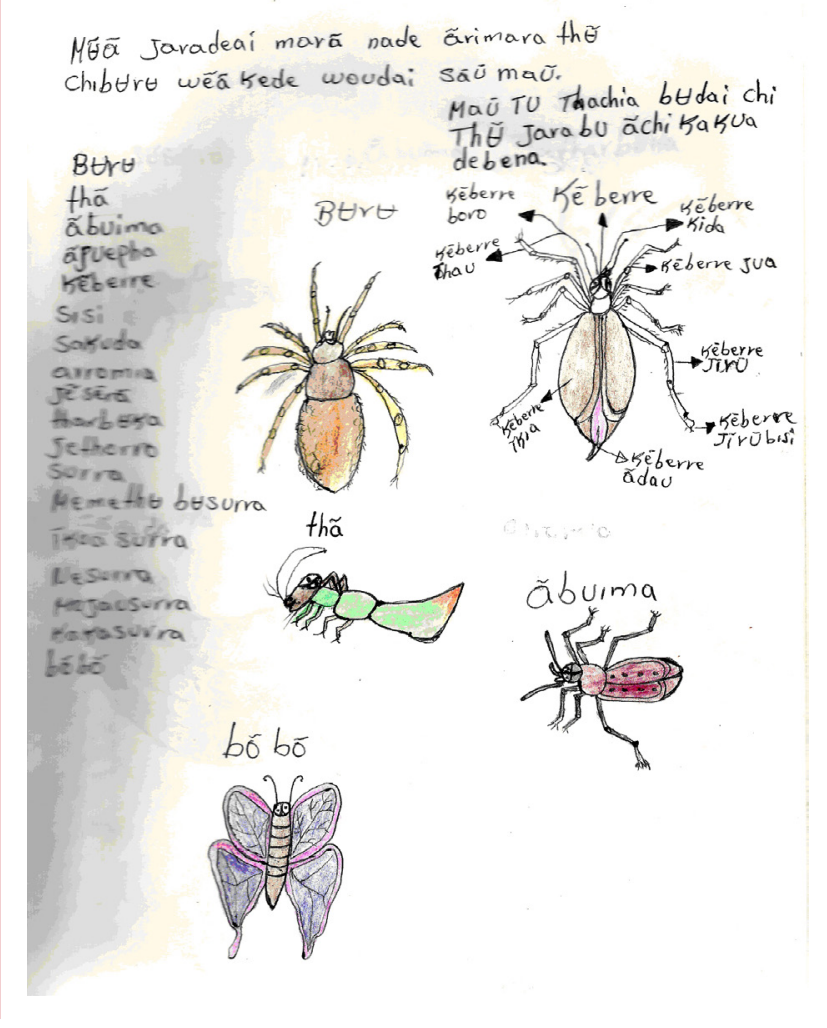

Mediante esta actividad se pretendía impulsar la producción de textos escritos por parte de docentes emberas y recoger cualquier tipo de producción en dicha lengua. Se trata de un diario que cada maestro debía desarrollar, en el que tenían que apuntar sus avances diarios y en el que tenían que recoger las tareas que se les encomendaban. Los objetivos básicos de esta actividad son el desarrollo de la escritura en embera y la producción de textos en dicha lengua que vaya más allá de confección de listas de palabras. En la figura 4 se puede observar una página del ewaritsa bupatat de uno de los docentes embera participantes en el curso. En la figura se observa una relación de nombres de insectos y los nombres de las partes del cuerpo de uno de ellos, pero también una frase que describe la definición de insecto. 


\section{FIGURA 5}

Portada del boletín Tachi pedea, el primero escrito exclusivamente en embera en el Chocó
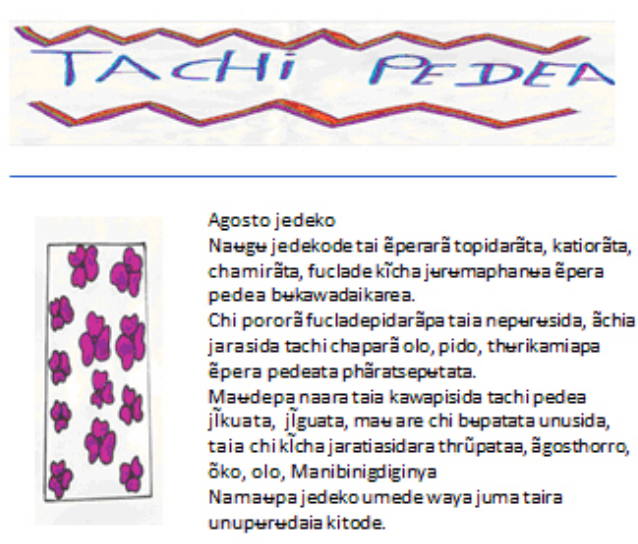

TAI KATIO PIDA KARI

TAI EPERA KATIO

MUKIRAPA MUKIRAPA

$\checkmark$ DEPA TUPATAMA

IBANACHAKE IBANACHAKE

PAKURUDE GTHG BAKEDE

WERACHAKE WERACHAKEPA

ECHAKE ATHAUKERAPARI

MUKIRAKADE MUKIRAKADE

PI-IA KAPHIPHIAPHEDA

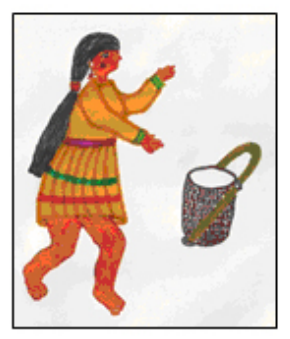

Uno de los talleres del curso se destinó a la confección de un boletín escrito exclusivamente en lengua embera para divulgar noticias acerca del curso y del camino emprendido por la lengua en la escritura. Se trata del primer boletín editado en embera y escrito por los propios embera. Además de promover la redacción de textos, también persigue la divulgación de la labor realizada y sensibilizar a toda la sociedad del Chocó de que es posible escribir en dicha lengua, tanto a los emberahablantes como a los desconocedores de la lengua. En la figura 5 se puede observar la primera página del primer Tachi pedea ('nuestra palabra', en castellano) escrito en agosto de 2012.

\section{Retos actuales}

Las comunidades con lenguas minorizadas en los países empobrecidos constituyen un caso especialmente vulnerable, en especial en lo referente a los sectores más desfavorecidos, entre los que hay que destacar a las niñas y a las mujeres. Asimismo, la educación, que constituye uno de los factores determinantes del desarrollo, si no tiene en cuenta la lengua propia, puede convertirse en un elemento añadido de discriminación para aquellas comunidades en peor situación. La lengua es uno de los principales motivos del fracaso escolar en los países con menos recursos (UNESCO, 2012). 
La formación de las nuevas generaciones en una lengua hegemónica que erradica la propia comporta cambios estructurales en su forma de entender el mundo y pone en riesgo la existencia de formas ancestrales de convivencia. Consideramos que la formación en la lengua propia no impide el aprendizaje del castellano u otras lenguas, ya que el hecho de convertirse en bilingües o plurilingües es, sin duda, un objetivo muy importante para el desarrollo personal y social de cualquier individuo. Además, la formación en la lengua propia facilita las posibilidades de acceso al conocimiento desde la propia cultura de cada comunidad indígena.

Desde el 2011, el proceso de planificación lingüística del embera no ha dejado de dar frutos: la alfabetización de hablantes adultos, la sensibilización, la creación de materiales didácticos y multimedia, la formación de docentes, las traducciones en esta lengua y la introducción de la misma en la educación primaria (en los departamentos de Antioquia y Chocó), pero está todavía lejos de alcanzar niveles mínimos que garanticen la supervivencia y normalización de la lengua.

El proceso de revitalización lingüística emprendido por los embera en los departamentos colombianos de Chocó y Antioquia deberá afrontar y superar numerosos retos. Con un uso escrito más generalizado del embera que amplíe los ámbitos de uso de la lengua, la convergencia de las dos propuestas ortográficas existentes en una propuesta común de consenso y la elaboración de un modelo de lengua estándar serán, seguramente, necesidades que aflorarán en el seno de la comunidad lingüística en un futuro próximo. Asimismo, resulta totalmente necesario que las leyes y las instituciones colombianas posibiliten y faciliten a la comunidad embera y a las comunidades indígenas, en general, la creación de su propia escuela y la gestión completa de la misma. Las leyes y las instituciones colombianas ya han dado pasos muy importantes en este sentido, pero todavía las limitaciones existentes son grandes. La creación y consolidación del sistema escolar indígena con un modelo plurilingüe que priorice la inmersión lingüística en lengua embera, junto con la capacitación de los docentes en didáctica bi-/plurilingüe en lengua minorizada, resultan esenciales.

Los indígenas han vivido siglos de políticas y prácticas sociales que les han impuesto modos de actuar colonialistas y discriminatorios. En la medida en que el pueblo embera camine en la normalización e incorporación de su lengua y cultura a la sociedad colombiana mejorará la cohesión de la sociedad embera y su bienestar social. La aportación de todas las lenguas y culturas es imprescindible para lograr un desarrollo equilibrado de la humanidad.

\section{Bibliografía citada}

Aguirre Licht, Daniel, 1992: "Previsibilidad del acento Embera-Chamí” en Lenguas Aborígenes de Colombia. Memorias 2, Bogotá: Universidad de los Andes - CCLA, 31-62.

Aguirre Licht Daniel, 1993: “Lenguas sobrevivientes del Pacífico colombiano” en Colombia Pacífico. Tomo I, Bogotá: FEN - Universidad Nacional de Colombia, 310-325. 
AguirRe Licht, Daniel, 1995: "Fonología del êbêra-chamí de Cristianía (Departamento de Antioquia)" en Lenguas Aborígenes de Colombia. Descripciones 8. Estudios fonológicos del grupo Chocó, Bogotá: Universidad de los Andes - CCLA, 9-86.

Aguirre Licht, Daniel, 1999: Embera, München: Lincom Europa.

Aguirre Licht, Daniel, Rubén Gonzalez Velez y Macario Panchi Carupia, 2013: Karta ēbēra bedea bu kawabiy ita / Manual de enseñanza y escritura ēbēra-chamí, Medellín: Gerencia Indígena, Gobernación de Antoquia (Colombia).

Arellano, Ignacio y Gabriel Arellano (eds.), 2002: El idioma katío. Ensayo gramatical, Frankfurt am Main - Madrid: Vervuert-Iberoamericana.

BorjA, Luis, A., 2009: Vocabulario embera eyábida. Con traducción al español. Un aporte a la educación y conservación de la cultura, Universidad Autónoma Latinoamericana, manuscrito no publicado.

Cayo Atienza, Ángel, 2002: El idioma katío. Ensayo gramatical, Frankfurt am Main - Madrid: Vervuert - Iberoamericana.

Chaves Mendoza, Álvaro y otros, 1992: Región del Pacífico: Awa-Cuaiquer-Emberá-Cuna-Waunana, Bogotá: Instituto Colombiano de Cultura Hispánica.

FABRE, Alain, 2005: Diccionario etnolingüístico y guía bibliográfica de los pueblos indígenas sudamericanos [http://www.ling.fi/Diccionario\%20etnoling.htm, fecha de consulta 18 de mayo 2016].

GARABIDE, 2010: La experiencia vasca. Claves para la recuperación lingüística e identitaria, Eskoriatza: Asociación Garabide.

Hoyos Benitez, Mario E., 2000: “Informe sobre la lengua embera del río Napipí" en María S. González de Pérez y María L. Rodríguez de Montes (eds.): Lenguas indígenas de Colombia: una visión descriptiva, Bogotá: Imprenta Patriótica Instituto Caro y Cuervo, 73-83.

Llerena Villalobos, Rito, 1992: "Estructura y variación en las fonologías de las lenguas êpêra de occidente y oriente" en Memorias del II Congreso del CCELA, Bogotá: Universidad de Los Andes.

Llerena Villalobos, Rito, 1994: "Sintaxis de la predicación de la lengua epera oriental del Alto Andágueda”, BIFEA 23(3), 437-462.

LoEwen, Jacob, A., 1963: "Chocó I: Introduction and Bibliography", International Journal of American Linguistics XXIX 3, 239-263

LÓPEZ, Luis E., e Inge SichRA, 2008: "Intercultural bilingual education for indigenous peoples in Latin American" en Jim Cummins y Nancy H. Homberger (eds.): Bilingual Education, vol 5. de Encyclopedia of Language and Education, New York: Springer, 295-309. 
MARTI, Félix, y otros, 2005: Words and worlds. World Languages Review, London: Multilingual Matters.

Mecha, Baltazar, Andoni Barreña y Mikel Mendizabal, 2013: "Enberera hizkuntza Kolonbian: ahozkotasunetik idazterako bidean”, BAT Soziolinguistika Aldizkaria 86, 159-175.

Mortensen, Charles A., 1999: A Reference Grammar of Northern Embera Languages. Texas: SIL International.

Nettle, Daniel, y Suzanne Romaine, 2000: Vanishing voices: the extinction of the world's language, Oxford: Oxford University Press.

PARdo, Mauricio, 1985: Gramática del idioma embera. Dialecto Alto Baudó. Informe final, Bogotá: Fundación Segunda Expedición Botánica.

PARDo, Mauricio, 1986: "La situación de la lingüística sobre el grupo Chocó", Memorias ICFS 42, 87-117.

PARDo, Mauricio, 1987: Elementos gramaticales del embera del noroccidente antioqueño, Medellín: Secretaría de Educación y Cultura de Antioquia (Colombia).

Pardo, Mauricio, y Daniel Aguirre, 1993: “Dialectología Chocó” en María L. Rodríguez de Montes (ed.): Estado actual de la clasificación de las lenguas indígenas de Colombia, Bogotá: Instituto Caro y Cuervo, 269-312.

Pinto García, Constancio, 1978: Los indios katíos: su cultura, su lengua, Medellín: Compas.

Rojas Curieux, Tulio, 2009: "Aspectos generales de la Llanura Costera del Pacífico" en Inge Sichra (coord.): Atlas sociolingüístico de pueblos indígenas en América Latina. Cochabamba: FUNPROEIB Andes, 647-676.

Sichra, Inge (coord.), 2009: Atlas sociolingüístico de pueblos indígenas en América Latina, Cochabamba: FUNPROEIB Andes.

UNESCO, 2012: Why language matters for the Millennium Development Goals, Bangkok: UNESCO Bangkok. 